Throughout the following year the number of children not suffering from severe respiratory infection was 1.5 times higher, than in the control group ( $p=0.0002)$. In a year's time in the core group the number of severe bronchitis cases reduced by $85.7 \%$ and no non-invasive pneumonias were recorded. In the control group the cases of diseases mentioned above reduced by $32.5 \%$ and $50 \%$ correspondently. Significantly less rarely - by $82 \%$ and $44.3 \%$ did acute otitis media occur. In 12 months' time symptoms of chronical tonsillitis definitely reduced by $89.2 \% \square 41.6 \%$, relapses of chronical adenoiditis were only recorded in $22.9 \% \square 37.6 \%$ of research participants. It is stated that the frequency of severe bronchitis $(\mathrm{p}=0.0186)$, severe otitis $(\mathrm{p}=0.0128)$, tonsillitis relapses $(\mathrm{p}$ $=0.0189)$ and adenoiditis $(\mathrm{p}=0.0423)$, definitely differed in groups of children only vaccinated with PCV13 and prepared for vaccination using $\alpha 2 b$-interferon.

Conclusions The usage of recombinant $\alpha 2 b$-interferon for prevaccination preparation allows us to conduct PCV13 vaccination in children suffering from recurrent respiratory infections more efficiently thus providing non-specific and specific protection of children's organisms. The data obtained clearly demonstrate that the usage of $\alpha 2 b$-interferon as a non-specific remedy for severe respiratory infections proves safe and efficient.

\section{P537 OBSTRUCTIVE SLEEP APNEA IN PRADER-WILLI SYNDROME: IS IT A HIDDEN CRISIS?}

${ }^{1}$ Anitha Sokay*, 'Lana Altabari, ${ }^{1,2}$ Edna Roche, ${ }^{1,2} \mathrm{M}$ Nadeem, ${ }^{1,2}$ Peter Greally. ${ }^{1}$ Tallaght Hospital, Dublin, Ireland; ${ }^{2}$ Trinity College Dublin, Dublin, Ireland

\subsection{6/archdischild-2019-epa.871}

Introduction Sleep abnormalities are recognized complications of Prader-Willi Syndrome (PWS). The prevalence of sleep disorders has varied across studies with a reported range of OSA from $44 \%$ to $100 \%{ }^{1}$ In prvious studies, a link has been suggested between the risk of OSA in those with PWS and body mass index (BMI), growth hormone treatment $(\mathrm{GHT})^{2}$ and the genetic type. Therefore, we set to examine the incidence and management of OSA as well as the relationship with GHT in the Irish paediatric population.

Methods This is a retrospective chart review of patients with a diagnosis of PWS and OSA from 1999-2018. We looked at polysomnography (PSG) results, ENT evaluation and subsequent recommended interventions. We categorised the type of sleep disorder and intervention used. After which we followed up with a post intervention PSG examining efficiency of treatment. PSG results were interpreted by the Paediatric Respiratory physician at Tallaght University Hospital.

Results A total of 49 patients were identified in the 19 years period, 14 of which were excluded (1 died, 5 left the country, and 7 had limited data). Of the remaining 35 patients, twenty patients $(57 \%)$ were diagnosed with moderate/severe OSA. Of those with moderate/severe, 15 of 20 (75\%) were received GHT, of whom $5(33.3 \%)$ developed a worsening of OSA. Eight of 35 patients (22.8\%) patients had a normal PSG, of whom one $(12.5 \%)$ developed OSA after initiating GHT. Seven (20\%) patients experienced equivocal PSG results. Notably, four of $7(57.1 \%)$ patients with equivocal polysomnogray results developed OSA, of whom one wasn't on GHT(25\%).
Of the 35 patients adentonsillectomy was recommended in 6 (17.1\%).

Conclusion In the paediatric Irish population with PWS, the overall incidence of OSA was $71.4 \%$. Our study confirmed that all children with PWS who are being considered for growth hormone therapy should primarily undergo assessment for OSA by polysomnography and should be referred for management by ENT for possible adenotonsillectomy. It also shows that despite ENT intervention or growth hormone therapy PSG remains a much needed screening for OSA in children with PWS.

\section{REFERENCES}

1. Sedky K, Bennett DS, Pumariega A. Prader willi syndrome and obstructive sleep apnea: co-occurrence in the pediatric population. J Clin Sleep Med. 2014;10 (4):403-409. doi:10.5664/jcsm.3616.

2. Nixon GM, Rodda CP, Davey MJ. Longitudinal association between growth hormone therapy and obstructive sleep apnea in a child with Prader-Willi syndrome. J Clin Endocrinol Metab. 2011;96(1):29-33. doi:10.1210/jc.2010-1445.

\section{P538 INCREASED SERUM LACTATE LEVELS ASSOCIATED WITH BETA-2 AGONIST USE IN PAEDIATRIC PATIENTS WITH ACUTE RESPIRATORY CONDITIONS}

John Travers, Basil Elnazir*. National Children's Hospital, Dublin, Ireland

\subsection{6/archdischild-2019-epa.872}

Introduction Beta-2 agonist use has been hypothesised to lead to increased lactate by the following mechanism: Increased lipolysis and free fatty acids from beta-adrenergic receptor stimulation inhibit Kreb's cycle conversion of pyruvate. Resultant elevated pyruvate is metabolised by anaerobic glycolysis, increasing lactate. ${ }^{1}$ There are few case reports of raised lactate from beta- 2 agonist treatment. We set out to identify any association of beta-2 agonist use and serum lactate levels in paediatric patients treated for acute respiratory conditions.

Methods We recorded the frequency of administering $2.5 \mathrm{mg}$ nebulised salbutamol and subsequent serum lactate levels for paediatric patients who presented to a hospital emergency department with respiratory distress and were referred to the respiratory specialist team over a 4 week period. Lactate levels were measured by arterial blood gas analysis within 2 hours of treatment. We also recorded age, gender and diagnosis.

Results 27 patients were admitted under respiratory team care following initial management, including salbutamol treatment, during the 4 week period. Mean age was 16 months and $46 \%$ were female. Conditions were diagnosed as bronchiolitis, lower respiratory tract infections, viral induced wheeze or exacerbations of asthma. $27 \%$ of patients had received three doses back-to-back, $65 \%$ had received single doses at 2 hour intervals and $8 \%$ had received single doses at 4 hour intervals. Lactate levels were elevated for all patients. Mean lactate was $2.2 \mathrm{mmol} / \mathrm{l}$. We did not identify any correlation between increasing frequency of administration and lactate level nor did we find any correlation between type of condition and lactate level. A review of patients showed that raised lactate was not as a result of other potential causes, such as tissue hypoxia, acute liver injury, diabetic ketoacidosis, ethanol toxicity, drug toxicity (including metformin, salicylates) or seizures. The raised lactate had no clear prognostic implication.

Conclusions We identified raised lactate levels in all paediatric patients that had been treated with nebulised salbutamol. This 
finding may be useful to clinicians to help avoid unnecessary use of salbutamol and to explain lactate findings when use of salbutamol is appropriately indicated.

\section{REFERENCE}

1. Sharif Z, Al-Alawi M. Beware of beta! A case of salbutamol induced lactic acidosis in severe asthma. Case reports 2018;2018:bcr-2017-224090

\section{P539 EVALUATION OF PSYCHOGENIC RESPIRATORY BEHAVIOUR IN CHILDREN}

${ }^{1}$ Abdurrahman Cahid Orengul, ${ }^{2}$ Hakan Yazan, ${ }^{3}$ Irem Yilmaz*, ${ }^{1}$ Erdem Ertas, ${ }^{2}$ Erkan Cakir. ${ }^{1}$ Bezmialem Vakif University, Faculty of Medicine, Department of Child and Adolescent Psychiatry, Istanbul, Turkey; ${ }^{2}$ Bezmialem Vakif University, Faculty of Medicine, Department of Pediatric Pulmonology, Istanbul, Turkey; ${ }^{3}$ Bezmialem Vakif University, Faculty of Medicine, Department of Pediatrics, Istanbul, Turkey

10.1136/archdischild-2019-epa.873

Introduction Psychogenic respiratory behaviours (PRB) such as psychogenic cough (tic cough), barking, sighing, feeling of shortness of breath, and throat clearing tic are common in adolescent children. These behaviours are difficult to diagnose and are usually diagnosed by psychiatric examination while excluding other diseases. Generally, symptoms disappear asleep. Prior to the diagnosis, these patients used many medications and they had many school absences. Therefore, early diagnosis is important. The aim of this study was to compare the sociodemographic and clinical characteristics of the patients with psychogenic respiratory behaviour to the control group and to evaluate their diagnosis according to DSM-5.

Method The study includes 52 children with PRB and control group, which includes 42 children, without any chronic disease. Patients who were followed-up in our pediatric pulmonology outpatient clinic between January 2017 and November 2018 were included in the study. Age, parental age, the presence of psychiatric disease in the family, family income and parental separation were questioned.

Results The mean age of the patients with PRB was $11.25 \pm$ 2.61 and it was $11.17 \pm 2.58$ in the control group. The rate of female patients was $48 \%(25 / 27)$ in the PRB group and $61.9 \%(26 / 16)$ in the control group. There were cough in 35 patients, shortness of breath in 11 patients, throat clearing in 11 patients and deep sighing in 6 patients (shortness of breath and deep sighing were together in most patients). Symptoms were more than 6 months in most patients. In $72 \%$ of the patients, there was a disappearance of symptoms in sleep. In addition, the disappearance of symptoms was observed at the rate of $25 \%$ in the school, $29.2 \%$ in social activity, $66.7 \%$ in the exercise. Considering the previous examinations; PA-AC radiography $84.6 \%$, allergy skin tests $76.9 \%$, blood test $82.7 \%$, spirometry $73.1 \%$ and CT $23.1 \%$ were performed in patients. At least one psychiatric disease was detected at $55.8 \%$ of PRB group and this rate was $28.6 \%$ in the control group. In the $\mathrm{PRB}$ group, attention deficit hyperactivity disorder, tic disorder and specific phobia were other common psychiatric.

Discussion and conclusion Psychogenic respiratory problems are usually diagnosed by exclusion of other diseases. This diagnosis should be considered in the adolescent age group which has no pathology in physical examination but especially high stress factor. In suspected patients, early psychiatric evaluation is important for early diagnosis of these patients.

\section{P540 OUTCOMES OF GIVING THE HERBAL BASED MEDICINE TONSILGON H TO PRE-SCHOOL CHILDREN WITH ADENO-TONSIL PATHOLOGY}

Vera Vavilova*, Alexandr Vavilov, Anastasia Cherkaeva, Irina Cherkaeva. HEKSMU, Kemerovo, Russian Federation

\subsection{6/archdischild-2019-epa.874}

Aim To study the efficiency and safety of the herbal preparation Tonsilgon $\mathrm{H}$ in clinical experience in pre-school children with adeno-tonsil pathology.

Materials and methods Within the period between 2013-2017 1876 children (2-5 years old), with nasopharyngeal and palatal tonsils pathology in case history, suffering recurrent respiratory infections were observed. The average age of the observed was $3,54 \pm 2,81$ years old. The pre-schoolers of the core group $(n=940)$ were given the herbal preparation Tonsilgon $\mathrm{H}$ in the form of drops dosed according to the age, Following the instructions during 30 days. The control group were made up from 936 children. The efficiency and safety of the medicine were analyzed before and after the course.

Outcomes A year before the course of treatment started $72.3 \%$ of children in the core group and $74.8 \%$ in the control group were suffering from nasopharyngeal tonsil hypertrophia 2 stage accompanied by adenoiditis $(p=0.2296)$. In a year's time after rehabilitation treatment only $31.6 \%$ of patients in the core group didn't show clinical performance improvement $(p=0.000)$. By the end of the research $64.6 \%$ of patients given the herbal preparation, proved reactivated nasal breathing $(\mathrm{p}=0.001)$; $58.3 \%$ of patients shew adenoiditis symptoms almost compensated $(\mathrm{p}=0.000) ; 86.5 \%$ proved the nasopharyngeal tonsil reduced from 2 to 1 stage $(\mathrm{p}=0.000) ; 78.9 \%$ shew rhinoscopic picture normalized $(\mathrm{p}$ $=0.000)$; endoscopic check proved palatal tonsils size reduction in $65.8 \%$ пациентов ( $p=0.000) ; 29.2 \%$ didn't even mention throat discomfort and irritation ( $\mathrm{p}=0.000)$; only $2.8 \%$ of children complained about swallow difficulties ( $\mathrm{p}=$ 0.000). The severity of nasopharyngeal and palatal tonsils hypertrophia in children not given the herbal preparation didn't change and even increased up to $83.6 \%$. What's more, the research proved the decrease in frequency of recurrent respiratory infections from 5-9 times a year to 2-3 times a year in $77.9 \%$ of children in the core group, as well as shortening the period of acute respiratory infections symptoms by 3 days, compared to previous cases ( $p=0.000)$.

Conclusion The research outcomes proved the efficiency and tolerability of the herbal preparation of combined effect Tonsilgon $\mathrm{H}$. Prospective observation of children given the herbal preparation as a rehabilitation remedy proved its effective influence on respiratory tract immunity formation. The obtained data allow us to recommend including the preparation Tonsilgon $\mathrm{H}$ into programs for immunity rehabilitation and prevention of adeno-tonsil pathology recrudescence in children from 1 year old. 